\title{
Oncothermia-booster (targeted radiofrequency) treatment -in some non-oncological diseases as special physiotherapy
}

\begin{abstract}
Introduction: Oncothermia (OTM) is based on electromagnetic interactions with the living organism. Its nano-targeting approach [established a newer paradigm, which could be applied not only in case of malignancies but in any other diseases, when the non-selective conditions are existing. This technique by now is well proven from the simple laboratory level to the several different clinical applications. Oncothermia method ignites the natural processes to rescue them from the system, re-establishing the better communication harmony between the cells of organism. This method will lead us to the treatment of some non-malignant diseases too to try delay their development or offer earlier recovery. Our aim was to use OTM on the common basis of equilibrium demand; and use the recognition of the deviations from the complex harmony of the organism or its part for selection to act properly.
\end{abstract}

Study protocol and method: Oncothermia was successfully applied for non malignant conditions like Lyme disease, for non-specific low-back pain, for Peyronie disease, and for Dupuytren's contracture, too. We made more extended study, proving in details the applicability of the OTM in these diseases, especially in the situations when traditional Chinese Medicine (TCM) is also applicable (acupuncture, permanent needle techniques). Our special permanent acupuncture method was proven previously and well fits to the complementary applications.

Results and discussion: The synergy of the ancient knowledge - application of heat energy - and the high-tech state-of-art of the medical knowledge could be established with our research. Recognition of the distortions in the healthy tissue has some common principles and possibilities in TCM and OTM: the left complexity of the living organization is recognized by both the methods. OTM application is a useful, harmless additional complementary treatment for management of selected diseases. Our topic is giving western trained physician's clinical applications of modern (Oncothermia-Booster) as a physiotherapeutic- equipment to accommodate accelerating interests in modern complex treatment of chronic low back pain and Dupuytren's contracture.

Conclusion: In recent study data verified the relevant end-points of the study: the safety, the quality of life (QoL), the shortened rest time, duration of painless state, cost/benefit ratio.

Keywords: physiotherapy, oncothermia, dupuytran's contracture, non-specific low back pain
Volume 6 Issue 3 - 2017

\author{
Agnes Mate, Istvan Molnar, Gabor Petrovics, \\ Gabriella Hegyi \\ Department of CAM, Pecs University, Hungary
}

Correspondence: Gabriella Hegyi, Department of CAM, Pecs University, Doctoral School, Hungary, Pecs,Vörösmarty u.4.Hungary, 7646, Hungary, Email drhegyi@hu.inter.net

Received: January 24, 2017 | Published: April 13, 2017
Abbreviations: OTH, oncothermy; LBP, low back pain; TCM, traditional chinese medicine; VAS, visual analog scale; QoL, quality of life

\section{Introduction}

Oncothermia (OTM) is based on electromagnetic interactions with the living tissue. ${ }^{1}$ Its nano-targeting approach ${ }^{2,3}$ establishes a new paradigm, which could be applied not only in case of malignancies but also in other diseases, when the non-selective conditions ${ }^{4}$ are valid. Oncothermia method ignites the natural processes to eliminate them from the system reestablishing the communication harmony between the cells. Our aim is to use OTM on the common basis of equilibrium demand; and use the recognition of the deviations from the complex harmony of the organism or its part for selection to act properly. This method will lead us to the successful treatment of some non-malignant diseases, too. This technique is well-proven from the many kind of the laboratory level to the clinical applications. ${ }^{5}$

\section{Method and procedure}

We decided to apply Oncothermia in the following 2 group of nonmalignant disease

\section{Dupuytren's contracture}

Dupuytren's Contracture (also known as Morbus Dupuytren's, or Dupuytren's disease and slang terms "Viking disease" or "Celtic hand"), is a fixed flexion contracture of the tenor due to a palmar fibromatosis. The fingers tend to move towards the palm and cannot be fully extended (straightened). Most commonly affected the ring finger and little finger. The middle finger may be affected in some more advanced cases, but the index finger and the thumb are not affected as frequently. These diseases sorted to the inherited proliferative connective tissue disorder that involves the hand's palmar fascia. It is called after Baron Guillaume Dupuytren, he was the first who described a solution of the operation to correct the affliction, and it was published in the Lancet in 1831. The progress of Dupuytren's 
contracture is slowly. Often is accompanied by less or more aching and itching. In this condition, the palmar fascia of patients thickens and shortens so that the tendons connected to the fingers cannot move freely. Incidence of the disease increases after age 40; at this age, men are affected more often than women. The progressive situation can be improved by operation in stage No, N1 and other alternative therapies can be tried, like Quercetin, Bromelain, DMSO, MSM, Acetylcarnitin $\mathrm{HCl}$, PABA, Nattokinase, Vitamin E (1940) Copper, Vitamin C, Massage. The palmar fascia becomes hyperplastic and contracts by time to time.

\section{Non-specific Low back pain}

Non-specific Low back pain (LBP), also known as lower back pain or lumbago, is a common disorder involving the muscles and bones of the back, but sometimes the lumbar nerve is affected. Low back pain may be classified by duration as acute (pain lasting less than 6weeks), sub-chronic (6 to 12 weeks), or chronic (more than 12 weeks). The condition may be further classified mechanical, non-mechanical, or referred pain. In most episodes of this condition, a specific underlying cause is not identified or even looked for, with the pain believed to be due to mechanical problems such as muscle or joint strain. If the pain does not go away with conservative treatment further testing may be needed to look for a more serious underlying problem. In most cases, imaging tools - X-ray computed tomography- are not useful and carry their own risks. Some low back pain is caused by less or more damaged intervertebral discs, and the" Lasegue test" is useful to identify this cause. In those with chronic pain, the pain processing system may malfunction, causing large amounts of pain in response to non-serious events. Despite this, the use of imaging in low back pain has increased. The treatment of acute non-specific low back pain is typically with conservative measures at reumatological outpatient surgery or General Practice (GP) consultation: using of some wellproved pain medications and the continuation of normal activity as the pain allows without bed rest. Chronic non-specific lower back pain needs more management for relieving permanent pain. The Booster, which is the unique instrument, was developed first for oncology, but can nevertheless also be successfully used in other medical fields in addition to oncology, in the follows: in fields such as rheumatology, sports medicine, neurology and neurosurgery, dermatology and analgesic therapy. We know that Oncothermia was successfully applied previously for Lyme-disease, ${ }^{6}$ for low-back pain, ${ }^{7}$ for Peyronie disease, ${ }^{8}$ and for Dupuytren's contracture, ${ }^{9}$ too. The number of patients was small one in the last mentioned study; therefore we made more extended study, proving in details the applicability of the OTM in these diseases, especially in the situations when traditional Chinese Medicine (TCM) is also applicable. Our special acupuncture method $^{10}$ well fits to the complementary applications. We enlarged number of selected patients suffering from Dupuytren's contracture in different stages of disease to investigate whether progression without side-effect could be stopped with elevation of QoL of patients and non-specific low back pain lasting more than 4 weeks where we used focused radiofrequency heat therapy as special physiotherapy.

The study for selected diseases was performed according to Helsinki Declaration and local ethical approval. Patient selection was made by rheumatologist outpatient's clinic. The period of study was 2012-2015.

We selected 76 patients suffering from Dupuytren's contracture in different stages (Figure 1). The selected patients were suffering from N0, N1, N2 stages received 2times/week special boosterphysiotherapy with Instrument (Booster, Oncotherm $\mathrm{GmbH}$, Germany), 30W, 25minutes, 1 course is 4 weeks ( 8 session), after $1 / 2$ years it was repeated 3times. The primer outcome measurement was "top of plate test", see Figure 2, painless extension of palm. Second outcome measurements were QoL, VAS of patients.

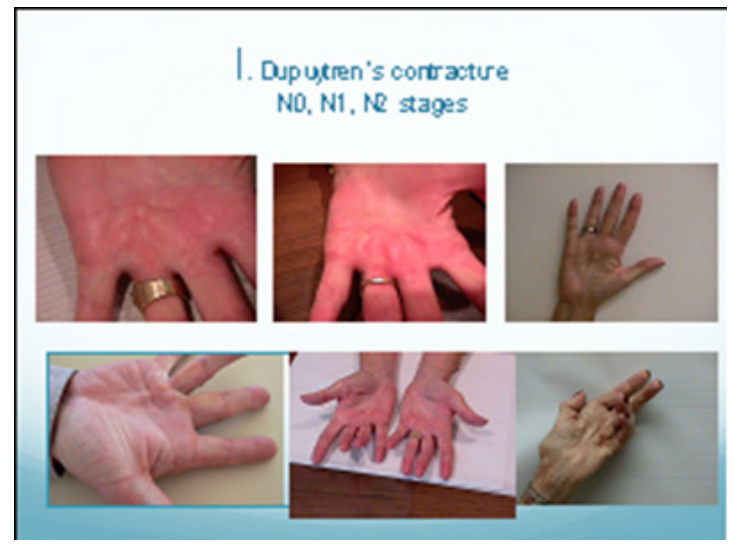

Figure I Dupuytren's contracture in different stages.

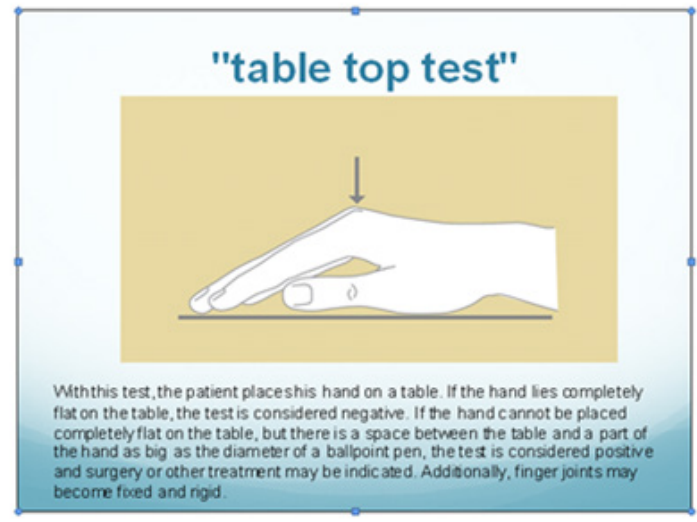

Figure 2 Table top test.

Also, we selected 155 patients suffering from non-specific low back pain, including verified ischialgy, discopathy, disc herniation and degenerative disease. Excluding criteria were ruptured disc herniation, caudal syndrome, malignancy-(bone metastasis from lung, breast, prostate, thyroid, among others), infectious (osteomyelitis; abscess) (Flow chart)

The selected patients received 2-times/week special Boosterphysiotherapy (with instrument Booster Oncotherm GmbH, Germany), $30 \mathrm{~W}$, duration was 25 minutes, 1 course is 4 weeks ( 8 sessions), and after $1 / 2$ years it was repeated 3 times. Additionally, all patients received meso therapy (neural therapy by local anesthetic Procain $0,5 \%, 2-3$ $\mathrm{ml} / \mathrm{session}$ and acupuncture (permanent monofilamentum insertion 1time / month according to TCM protocol and protocol of Neural therapy) Figures 3\&4. Questionnaire on pain and QoL was filled in all sessions during physical examination. Visual Analog Scale (VAS), "Top of plate test" was registered during the all period of treatments.

\section{Results}

We recognized in N0 and N1st stage of Dupuytren's cases significant delay of progression, moderately in $2^{\text {nd }}$, no changes in progressive stages after 2years follow-up. According to delayed progression in VAS can be seen result (Figure 5). The following additional therapies used to apply for Dupuytren's cases Quercetin, Bromelain, DMSO, MSM, Acetylcarnitin HCl, PABA, Nattokinase, Vitamin E (1940) Copper, Vitamin C, usual continuous massage therapy. 


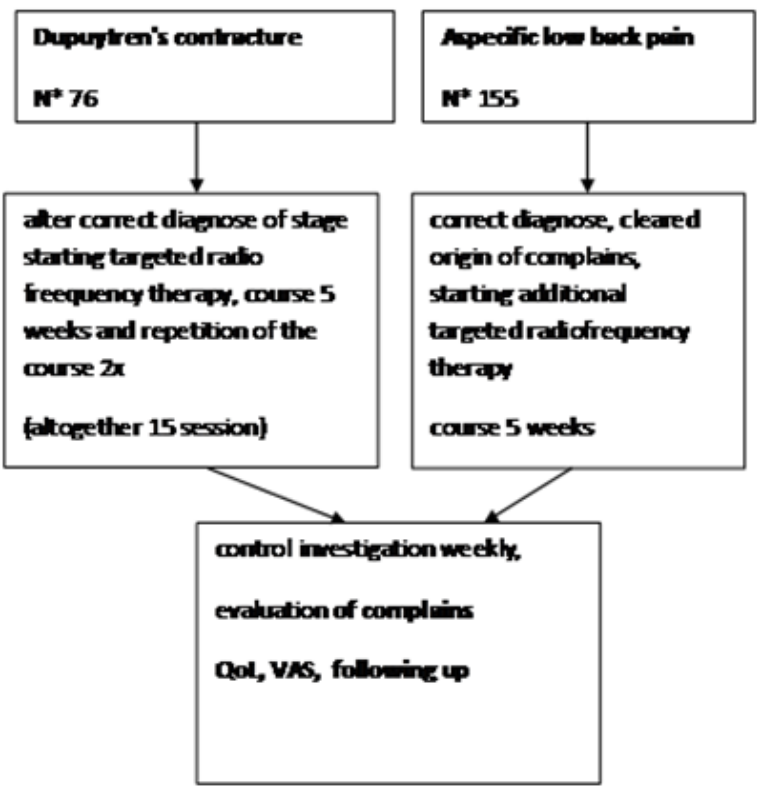

Flowchart for procedure

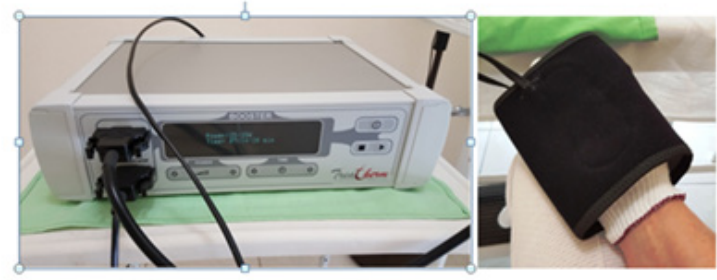

Figure 3 Course of treatment with Booster Instrument.

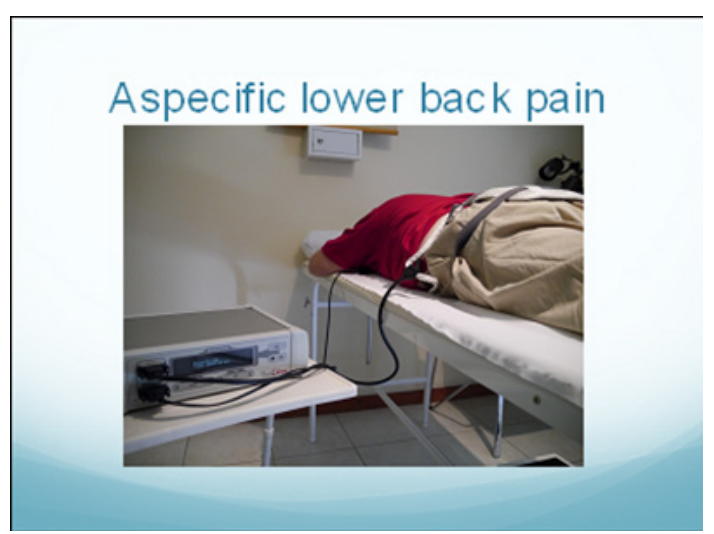

Figure 4 Low back pain treatment.

\section{Discussion}

The second investigated group was non-specific low back pain Better results were achieved in cases originated from ischialgy, chronic degenerative lumbar disc cases, low back pain caused by post-operative edema. The rest time out of work was less. The low back patents needed fewer intakes of analgesic pills (Figure 6).

According to our experience the "targeted radiofrequencyphysiotherapy as Booster" application is free of side effects (but skill, practice is necessary), harmless, well tolerable by patients, easy to apply by skilled administration. It is advised to consider that complementary treatment of selected diseases is valuable for introducing into daily rheumatologic practice. Also we recognized the less intake of medicaments in cases during sessions of low back pain, which is important to avoid unnecessary side effects of medicaments (NSAID).

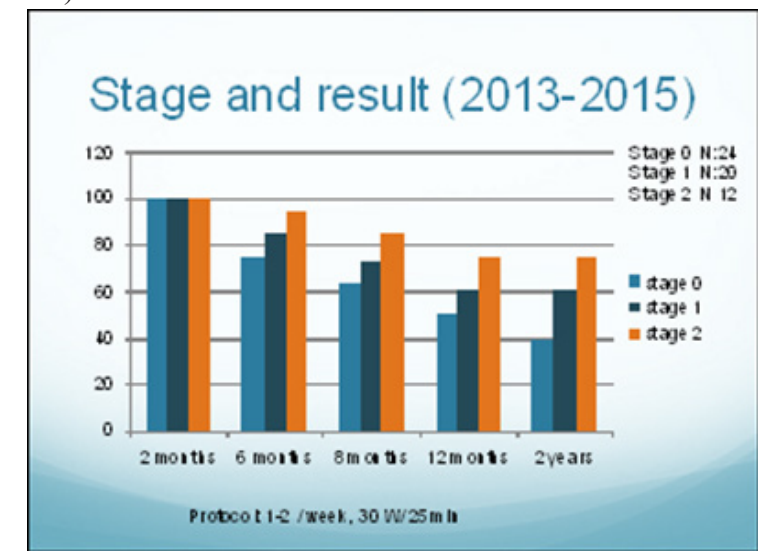

Figure 5 Results in Dupuytran's contracture.

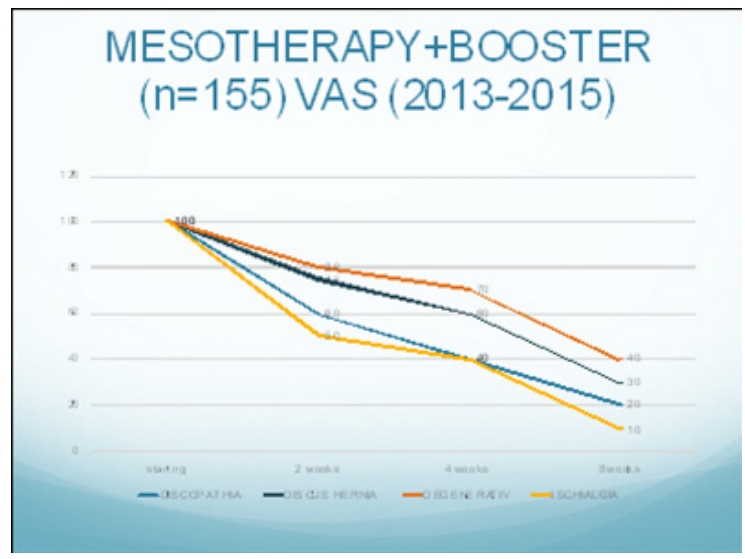

Figure 6 Results in low back pain.

\section{Conclusion}

The targeted radiofrequency therapy performed by Booster Instrument, is used as a supplement to physiotherapy, general used drug treatments. It is not advised to be a stand-alone treatment device, but should be used in a supporting role with drug treatments to enhance their effects. The purpose of usage of the Booster is to increase the blood flow in the treatment area. Selection at cellular level does not occur, only a heating of the deep layers of tissue in the region where the electrode is positioned. The deep-heating effect is a result of Joule loss and leads to vascular dilatation in the treatment area, that in turn, improves higher and better blood perfusion and thus the drugs and more oxygen are transported to the treated area. The temperature in this area is $37-39^{\circ} \mathrm{C}$ (moderate, classic Hyperthermia). This is the optimum temperature for the effect of booster. Further improvements are the follows: increased fibroblast activity and increased capillary growth, increased nutrient concentration and metabolic activity, synergetic increase in the field-dependent effects. It is leads to the optimization of membrane stimulation and activation of signal channels etc.). More recognized effects are: increased reactions to heat and field exposure (mainly the development of heat shock proteins, HSP), increased venous and lymphatic flow, which finally causes changes in the physical properties of the tissue.

Recognition of the distortions in the healthy tissue has some common principles and possibilities in TCM and OTM: the loss of complexity of the living system is recognized by both methods. The 
synergy of the ancient well-proved knowledge and the high-tech state-of-art of the medical knowledge (newer application of targeted radiofrequency could be established with this research. The positive result of this study was the recognition to apply the radiofrequency heat therapy for non-malignant, developing connective tissue diseases with positive result.

The limitation of this study is lack of double-blind control and lack of "sham" application. We need further researches in the future to clarify the exact undergoing process in selected disease group, but results show improvement and better QoL on regarded diseases, therefore it is advisable to use this physiotherapeutic method in daily practice of mentioned disease after careful selection. ${ }^{11,12}$

\section{Acknowledgments}

The present scientific contribution is dedicated to the 650th anniversary of the foundation of the University of Pécs, Hungary.

\section{Conflicts of interest}

Author declares there are no conflicts of interest.

\section{Funding}

None.

\section{References}

1. Szasz A, Szasz N, Szasz O. Oncothermia. Principles and Practices. Germany: Springer Verlag; 2010.

2. Szasz A. Electromagnetic effects in nanoscale range, chapter in book Cellular response to physical stress and therapeutic application. In Shimizu T \& Kondo T, Editors. Inc, USA: Nova Science Publishers, 2013.
3. Szasz O, Szasz A. Oncothermia - Nano-heating paradigm. J Cancer Sci Ther. 2014;6(4):117-121.

4. Szasz A. Challenges and solutions in oncological hyperthermia. Thermal Med. 2013;29(1):1-23.

5. Andocs G, Szasz O, Szasz A. Oncothermia treatment of cancer: from the laboratory to clinic. Electromagn Biol Med. 2009;28(2):148-165.

6. Zais O, Szasz A. Lyme disease and oncothermia, Conference Papers in Medicine. 2013;2013:1-3.

7. Hegyi G, Jian L, Szasz A. Low back pain-Complex approach of treatment by different CAM modalities, Conference Papers in Medicine. $2013 ; 2013: 1-8$

8. Ballerini M, Baronzio GF, Capito G, et al. Androtherm application for the Peyronie's disease. Conference Papers in Medicine. 2013;2013:1-6.

9. Hegyi G. Usage of Booster (oncothermia) in rehabilitation of nonmalignant conditions. Chin: 32nd Annual Conference of ICHS 2013, Panyu; 2013.

10. Hegyi G, Szigeti GP. Rehabilitation of Stroke Patients using Yamamoto New Scalp Acupuncture - A Pilot Study. J Altern Complement Med. 2012;18(10):971-977.

11. Kanodia AK, Legedza ATR, Davis RB, et al.Perceived benefit of Complementary and Alternative Medicine (CAM) for back pain: A national survey. Journal of the American Board of Family Medicine. 2010; 23(3):354-362.

12. Hegyi G, Szasz Oliver, Szasz Andras. Oncothermia: A New Paradigm and Promising Method in Cancer Therapies. Acupunct Electrother Res. 2013;38(3-4):161-197. 